\title{
Unravelling the effect of data augmentation transformations in polyp segmentation
}

\author{
Luisa F. Sánchez-Peralta ${ }^{1}$ (D) Artzai Picón ${ }^{2}$ (D) Francisco M. Sánchez-Margallo ${ }^{1}$ (D) J. Blas Pagador ${ }^{1}$ (D)
}

Received: 13 January 2020 / Accepted: 14 September 2020 / Published online: 28 September 2020

(c) The Author(s) 2020

\begin{abstract}
Purpose Data augmentation is a common technique to overcome the lack of large annotated databases, a usual situation when applying deep learning to medical imaging problems. Nevertheless, there is no consensus on which transformations to apply for a particular field. This work aims at identifying the effect of different transformations on polyp segmentation using deep learning.

Methods A set of transformations and ranges have been selected, considering image-based (width and height shift, rotation, shear, zooming, horizontal and vertical flip and elastic deformation), pixel-based (changes in brightness and contrast) and application-based (specular lights and blurry frames) transformations. A model has been trained under the same conditions without data augmentation transformations (baseline) and for each of the transformation and ranges, using CVCEndoSceneStill and Kvasir-SEG, independently. Statistical analysis is performed to compare the baseline performance against results of each range of each transformation on the same test set for each dataset.

Results This basic method identifies the most adequate transformations for each dataset. For CVC-EndoSceneStill, changes in brightness and contrast significantly improve the model performance. On the contrary, Kvasir-SEG benefits to a greater extent from the image-based transformations, especially rotation and shear. Augmentation with synthetic specular lights also improves the performance.

Conclusion Despite being infrequently used, pixel-based transformations show a great potential to improve polyp segmentation in CVC-EndoSceneStill. On the other hand, image-based transformations are more suitable for Kvasir-SEG. Problem-based transformations behave similarly in both datasets. Polyp area, brightness and contrast of the dataset have an influence on these differences.
\end{abstract}

Keywords Polyp segmentation · Deep learning · Data augmentation · Transformations · Semantic segmentation

\section{Introduction}

Deep learning techniques have been widely used for the last years as they have proved their ability to extract features for different computer vision tasks such as object detection, classification or segmentation [1]. Undoubtedly, these techniques have also been used for medical imaging with great

Electronic supplementary material The online version of this article (https://doi.org/10.1007/s11548-020-02262-4) contains supplementary material, which is available to authorized users.

Luisa F. Sánchez-Peralta

lfsanchez@ccmijesususon.com

1 Jesús Usón Minimally Invasive Surgery Centre, Road N-521, km 41.8, 10071 Cáceres, Spain

2 Tecnalia Research and Innovation, Zamudio, Spain success [2,3]. Even though, one limitation that must be faced in this field is the lack of large datasets with relevant annotations and/or labelling [4, 5]. One of the most widely used strategies for addressing this problem is data augmentation [6].

Data augmentation for images consists of increasing the amount and diversity of training cases based on the available images in the database through the application of image transformations such as translation or flipping of the original image [7]. Different computational libraries have been created to perform these transformation functions [8, 9]. However, the selection of the most suitable strategy remains a trial-and-error process that depends on the experience, imagination and time of the researcher [10]. There are several studies analysing the effect of data augmentation for image 
classification tasks [11-14], but this field is not fully explored for semantic segmentation yet [15].

Computer-assisted diagnosis (CAD) systems for early detection of colorectal cancer have also benefited from the application of deep learning techniques [16-18]. Publicly available datasets range from hundreds of images with a manually segmented binary mask, such as CVC-EndoSceneStill [19] or Kvasir-SEG [20], to thousands of video frames with an approximated elliptical binary mask, such as CVCVideoClinicDB [21, 22]. For polyp segmentation, it is easy to find several works in which data augmentation has been used. Nevertheless, there is a wide variety of transformations selected as well as their ranges (for example, rotating between $-45^{\circ}$ and $45^{\circ}$ instead of between $-90^{\circ}$ and $90^{\circ}$ ). Table 1 gathers the applied transformations and their ranges, when available, for recent works on polyp segmentation using deep learning. Although there are authors who do use data augmentation, they do not describe the transformations applied [23]. Besides, it is also important to point out that more intense data augmentation does not necessarily yield to increased performance [24]. The particularities of the medical image type must also be taken into consideration for selecting data augmentation transformations, as the image might have particularities that affect image processing methods. For polyp segmentation, specular lights negatively affect detection methods as they prominently appear, hiding colour and textural information [25].

We hypothesize that the application of different transformations as well as different ranges for the same transformation might lead to differences in performance. Thus, the objective of this work is to elucidate the effect of different image transformations and their ranges used for data augmentation for polyp segmentation. Therefore, this work does not pursue to obtain the best segmentation results but to analyse how the different transformations and their ranges used in data augmentation might influence the results of polyp segmentation in endoscopic images using deep learning.

\section{Methods}

\section{Transformations}

Different transformations have been considered in this study, which can be classified into three categories. For each transformation, a suitable range of values has been established (Table 2). Figure 1 shows an example of the result of applying each transformation to an image. In the case of image-based transformations, image and mask are transformed in the same way.

To model the specular lights, the CVC-EndoSceneStill database [19] has been used, as it provides a manually segmented class for specular lights in endoscopic images.

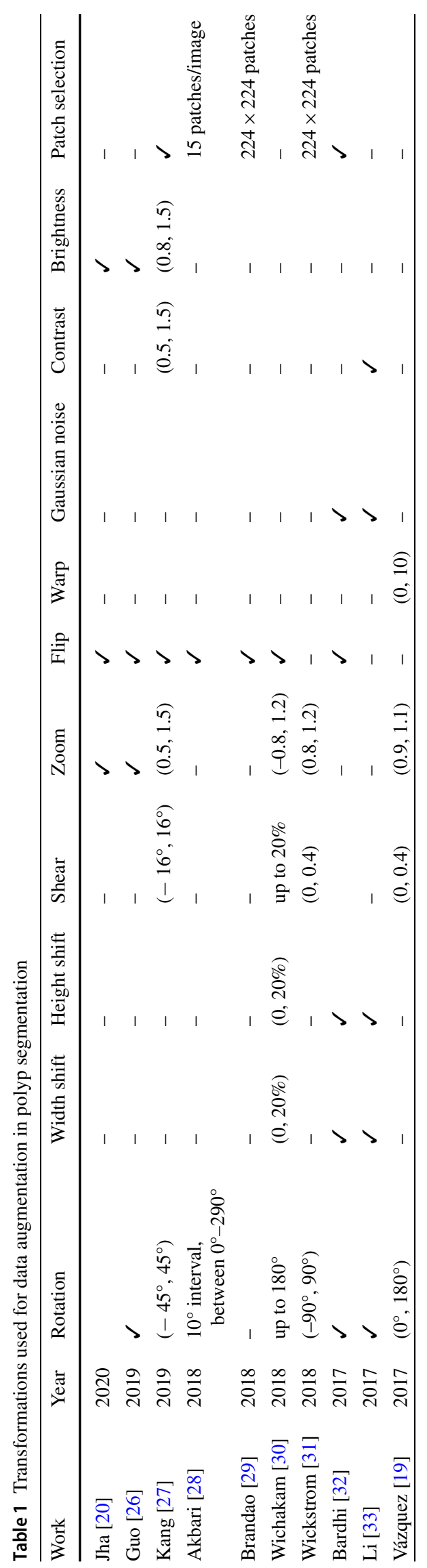


Table 2 Transformations and ranges analysed in this study

\begin{tabular}{|c|c|c|c|}
\hline Transformation & Parameter definition & Ranges & Total cases \\
\hline \multicolumn{4}{|l|}{$\begin{array}{l}\text { Image-based } \\
\text { transformations }\end{array}$} \\
\hline Width shift & $\begin{array}{l}\% \text { of the image displaced to } \\
\text { the right or to the left }\end{array}$ & $0-90 \%$, with $10 \%$ intervals & 9 cases \\
\hline Height shift & $\begin{array}{l}\% \text { of the image displaced up } \\
\text { or down }\end{array}$ & $0-90 \%$, with $10 \%$ intervals & 9 cases \\
\hline Rotation & $\begin{array}{l} \pm \text { Degrees that the image is } \\
\text { rotated }\end{array}$ & $\begin{array}{l}0-180^{\circ} \text {, with up to } 45^{\circ} \\
\text { intervals }\end{array}$ & 8 cases \\
\hline Shear & $\begin{array}{l} \pm \text { Shear angle in } \\
\text { counter-clockwise } \\
\text { direction }\end{array}$ & $\begin{array}{l}0-180^{\circ}, \text { with up to } 45^{\circ} \\
\text { intervals }\end{array}$ & 8 cases \\
\hline Zoom out & $\begin{array}{l}\text { Factor by which the image } \\
\text { size is multiplied }\end{array}$ & $\begin{array}{l}1-x, x \in[0.1,0.9] \text {, with } \\
0.1 \text { intervals }\end{array}$ & 9 cases \\
\hline Zoom in & $\begin{array}{l}\text { Factor by which the image } \\
\text { size is multiplied }\end{array}$ & $\begin{array}{l}1+x, x \in[0.1,1.0], \text { with } 0.1 \\
\text { intervals }\end{array}$ & 10 cases \\
\hline Flip & $\begin{array}{l}\text { Vertically and horizontally } \\
\text { flip the image }\end{array}$ & True & 2 cases \\
\hline Elastic deformation & $\begin{array}{l}\text { Parameters as indicated in } \\
\text { [32] }\end{array}$ & $\begin{array}{l}\alpha \text { values: } 250,500,1000, \\
2000,3000,4000,5000, \\
6000 \\
\sigma \text { value: fixed at } 40\end{array}$ & 8 cases \\
\hline \multicolumn{4}{|c|}{ Pixel-based transformations } \\
\hline Brightness & $\begin{array}{l} \pm \text { value to be added to the } \\
\text { actual pixel value for all } \\
\text { RGB channels equally }\end{array}$ & {$[25,175]$, with 25 intervals } & 7 cases \\
\hline Brightness & $\begin{array}{l}\text { Value to be added to the } \\
\text { actual pixel value for each } \\
\text { RGB channel } \\
\text { independently }\end{array}$ & {$[25,175]$, with 25 intervals } & 7 cases \\
\hline Contrast & $\begin{array}{l}\text { Value to multiply the actual } \\
\text { pixel value for all RGB } \\
\text { channels equally }\end{array}$ & $\begin{array}{l}{[1-x, 1+\mathrm{x} x, x \in[0.2,1.0]} \\
\quad \text { in intervals of } 0.2\end{array}$ & 5 cases \\
\hline Contrast & $\begin{array}{l}\text { Value to multiply the actual } \\
\text { pixel value for each RGB } \\
\text { channel independently }\end{array}$ & $\begin{array}{l}{[1-x, 1+x], x \in[0.2,0.8]} \\
\quad \text { in intervals of } 0.2\end{array}$ & 4 cases \\
\hline \multicolumn{4}{|l|}{$\begin{array}{l}\text { Application-based } \\
\text { transformations }\end{array}$} \\
\hline Specular lights & $\begin{array}{l}\text { Overexposed light ellipses } \\
\text { simulating the effect of } \\
\text { bright points }\end{array}$ & True & 1 case \\
\hline Blurry images & Window size of a mean filter & {$[1,15]$, only even integers } & 7 cases \\
\hline
\end{tabular}

Specular lights are modelled as ellipses of variable size and orientation. Size of major and minor axes are obtained from the specular lights in CVC-EndoSceneStill database, corresponding to a mean major axis of $7.77 \pm 10.36$ pixels (range $0-259.81$ ) and a mean minor axis of $3.82 \pm 4.29$ pixels (range 0-137.39). The number of specular lights per image is modelled as a positive left-skewed distribution, with mean 18.20 and standard deviation 16.97, according to the distribution of CVC-EndoSceneStill. In the image, pixel values are set to 255 in all channels to create the ellipses according to the previously described distributions, with random locations on the image.

\section{Datasets, architecture and training process}

Two publicly available datasets have been used in this work. CVC-EndoSceneStill [19] contains 912 images obtained from 44 video sequences collected from 36 patients. It explicitly indicates the images belonging to the training, validations and test sets. In this work, this division has been used. This way, all experiments use the same images, which allows for a fair comparison of performance. The training, validation and test sets comprise 547, 183 and 182 images, respectively. The second dataset is Kvasir-SEG [20]. It provides 1000 polyp images. The dataset has been divided into training, valida- 


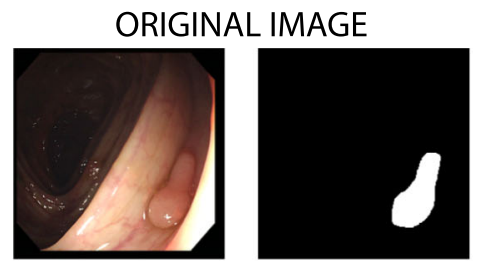

IMAGE-BASED TRANSFORMATIONS

Width Shift
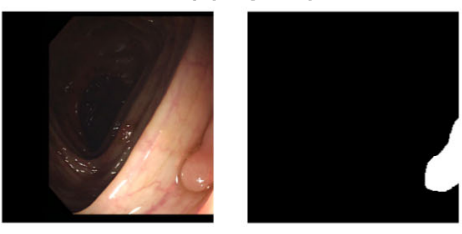

Shear

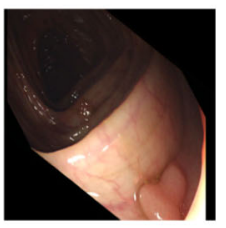

Horizontal Flip
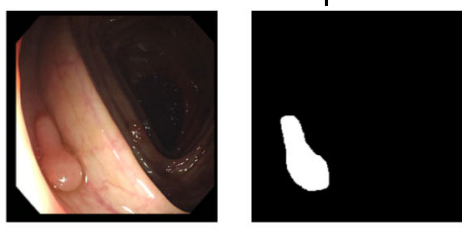

Height Shift

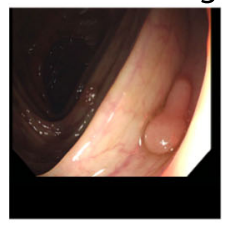

Zoom In
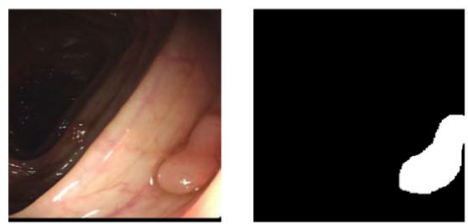

Vertical Flip
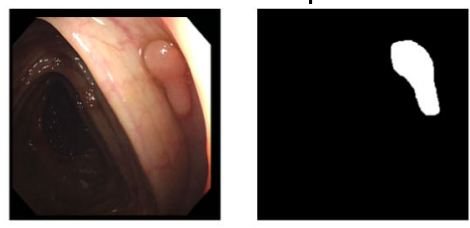

PIXEL-BASED TRANSFORMATIONS

Brightness

(all channels equally)

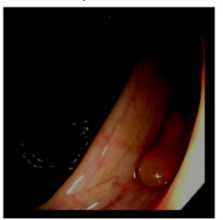

Contrast

(all channels equally)
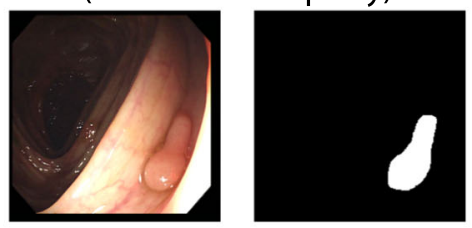

Brightness

(each channel independently)

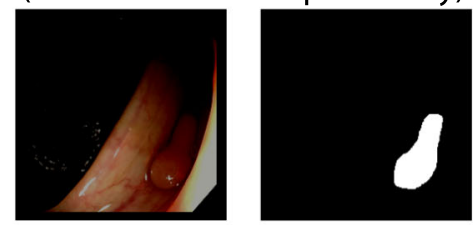

Contrast

(each channel independently)
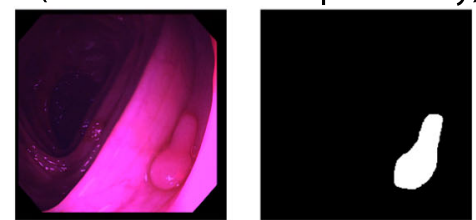

Rotation

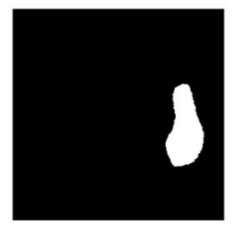

Zoom Out

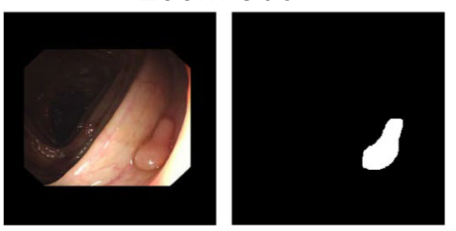

Elastic Deformation
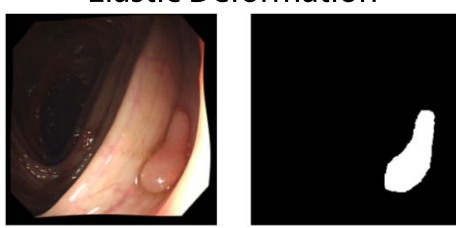

PROBLEM-BASED TRANSFORMATIONS

Specular Lights

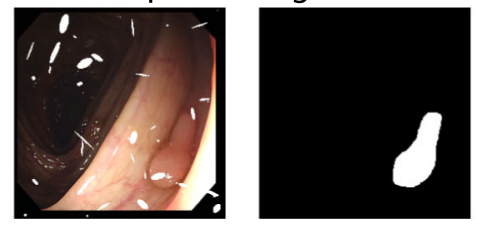

Blurry Frames

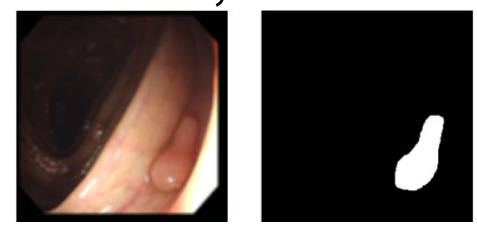

Fig. 1 Original and transformed images 
Table 3 Details for the datasets used in this study

\begin{tabular}{lcc}
\hline & CVC-EndoSceneStill & \multicolumn{1}{l}{ Kvasir-SEG } \\
\hline Void area (\%) & $23.73 \pm 5.57(27.83-14.62)$ & $15.23 \pm 4.82(28.44-6.16)$ \\
Polyp area relative to the valid area (\%) & $12.50 \pm 11.49(66.15-0.75)$ & $17.36 \pm 15.65(83.66-0.61)$ \\
Mean value of brightness channel in HSV & $0.560 \pm 0.006(1.000-0.000)$ & $0.622 \pm 0.003(1.000-0.000)$ \\
$\quad$ [34] & & \\
Histogram flatness measure [35] & $0.858 \pm 0.121(0.959-0.000)$ & $0.419 \pm 0.443(0.962-0.000)$ \\
Histogram spread [35] & $0.252 \pm 0.088(0.520-0.076)$ & $0.218 \pm 0.070(0.432-0.075)$ \\
\hline
\end{tabular}

Results are reported as mean \pm standard deviation. Minimum and maximum values are indicated between brackets. The void area refers to the black area in the images, while the remaining area is considered as valid area tion and test sets (800, 200 and 200 images, respectively), as this division is not provided by the dataset's owners. Both datasets provide binary masks for each polyp image, where pixels corresponding to the class are labelled with 1 , and 0 otherwise. Each dataset is used on its own to replicate the same experiments for further comparison of results. Table 3 shows some characteristics of the images included in the test sets of the datasets. Kvasir-SEG presents bigger polyps than CVC-EndoSceneStill, with images that are brighter and with more contrast and where the void area is smaller.

Our network architecture (Fig. 2) is based on a U-Net architecture [36]. The down-sampling path transforms the input image of size $256 \times 256 \times 3$ to a feature map of $16 \times$ $16 \times 1024$ by applying five convolutional blocks. These blocks consist of two $3 \times 3$ convolutional layers, each one with a rectified linear unit, and a $2 \times 2$ max pool layer, except for the last block. The up-sampling path includes four blocks that produce a $256 \times 256 \times 1$ probability map. Each block starts with a $2 \times 2$ up-sampling layer followed by a $3 \times 3$ convolutional layer, to whose result the corresponding feature map from the down-sampling path is concatenated. Zero padding preserves sizes along convolutional layers. We included batch normalization both in down- and up-sampling paths.

The network has been implemented using Keras [37] and Tensorflow [38] as backend. Experiments were run on a NVIDIA GTX 1080 GPU with 8 GB memory. The network has been pretrained using CVC-VideoClinicDB [21, 22], whose polyp masks are not precise but approximated to elliptical shapes. The datasets in "Transformations" section are then used to finetune this pretrained model with fixed parameters for all experiments:

- Adam optimizer, with default parameters in Keras: ams$\operatorname{grad}=$ false; beta $\_1=0.9$ and beta $\_2=0.999$

- Learning rate: starting at $10^{-4}$, decreasing to half each epoch and recovering to $10^{-4}$ each 5 epochs

- 15 epochs

- Batch size: 4

- Image input size: $256 \times 256 \times 3$

- Dropout: 0.5
Each experiment has been repeated ten times to minimize the effect of randomly applying transformations. Results are shown in terms of mean \pm standard deviation of the mean. A baseline level has been established by finetuning the model without applying any data augmentation.

Since semantic segmentation is performed through a pixel-wise classification, we face an unbalanced dataset where the negative class (no polyp) is more present than the positive one (polyp) in each image. Therefore, the selected loss function combines the binary cross-entropy and the Jaccard index as in [39]:

Loss $=-\frac{1}{n} \sum_{i, j}\left(y_{i, j} \log \hat{y}_{i, j}+\left(1+\hat{y}_{i, j}\right)\right) \log \left(1-\hat{y}_{i, j}\right)-\log J$

where the first term corresponds to the binary cross-entropy, being $y_{i, j}$ the ground truth class for pixel $(i, j)$ and $\hat{y}_{i, j}$ the predicted class; and $J$ is the Jaccard index or Intersection over Union (IoU) defined as a similarity measure between sets $A$ and $B$ as:

$J=\operatorname{IoU}(A, B)=\frac{|A \cap B|}{|A \cup B|}=\frac{|A \cap B|}{|A|+|B|-|A \cap B|}$,

where $|X|=\sum_{i} x_{i}$ being $x_{i}$ is the $\mathrm{i}$-th element of set $X$; $\cap$ is the intersection of sets and $\cup$ is the union of sets.

\section{Statistical analysis}

Results of the ten repetitions have been statistically analysed to identify differences between distributions, using $\mathrm{R}$ (version 3.6.1) and RStudio (version 1.2.5033). Permutation test [40] is selected as no assumption on the distributions is required. In the permutation test, firstly the "observed mean" is calculated as the difference between means for the baseline and the group under study. Data are then shuffled and randomly assigned to each group and the corresponding "calculated mean" is obtained as the difference between means of the two groups. After 10000 repetitions, the $p$ value is determined as the percentage of calculated means which are greater than the observed mean. Significance is evaluated at $p$ 


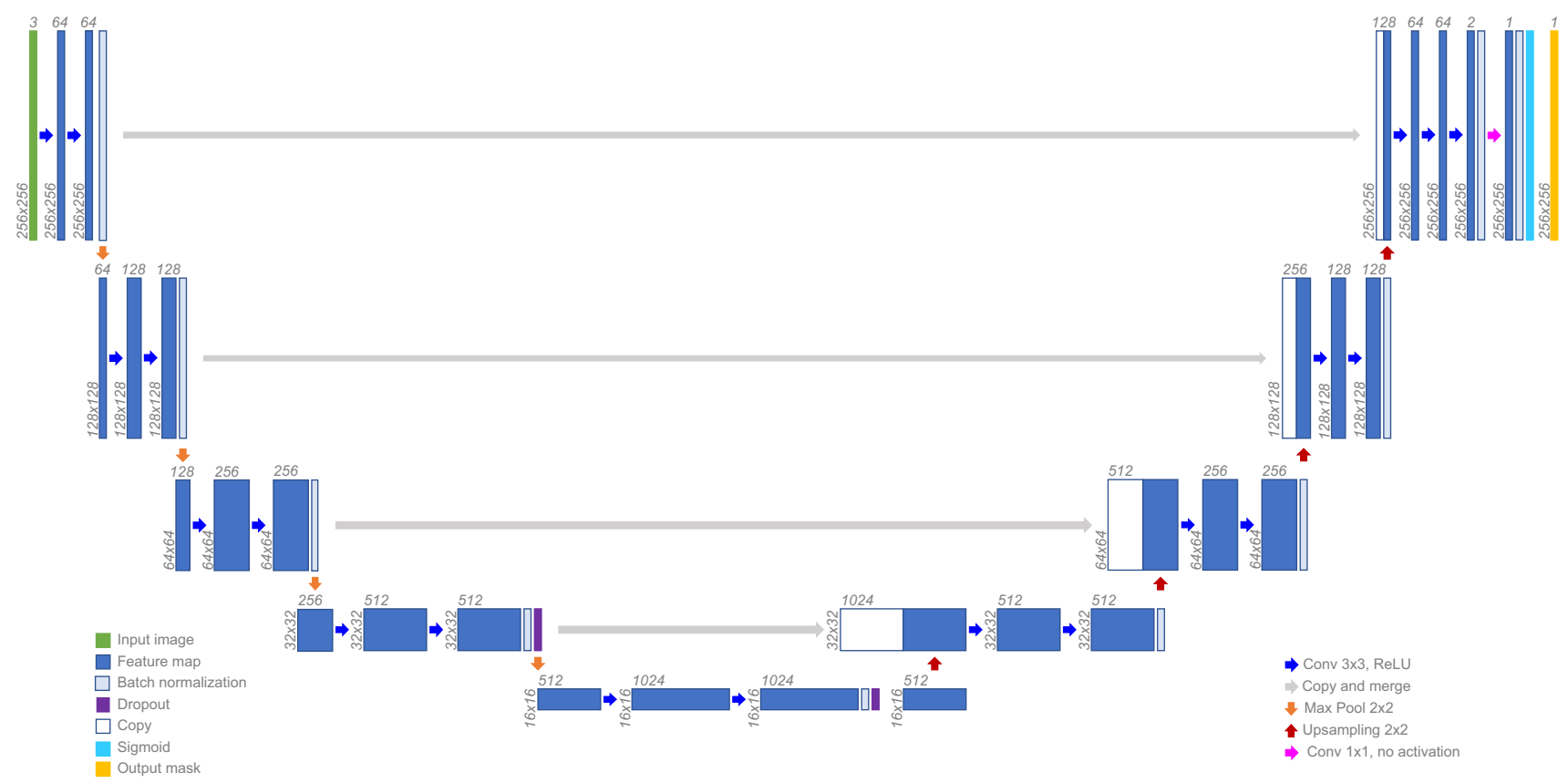

Fig. 2 Network architecture. Figure based on [36]

value $<0.05, p$ value $<0.01$ and $p$ value $<0.001$. This analysis is performed for each dataset independently.

\section{Results}

For both datasets, Table 4 shows the results for the baseline and all transformations and ranges, together with the results of the permutation test to establish statistically significant differences between baseline and transformations.

Figures 3, 4 and 5 show the range with the highest mean for each transformation for the CVC-EndosceneStill and KvasirSEG. Figures for all transformations and ranges can be found in the Supplementary material 1 for CVC-EndoSceneStill dataset and Supplementary material 2 for Kvasir-SEG. All figures show boxplots combined with violin plots, representing the distribution of the results. In these violin plots, the ideal outcome is that the distribution presents a peak at 1 . Therefore, the more the distribution looks alike this peak, the better the performance is.

Image-based transformations have different behaviours depending on the dataset, transformation and range. In first place, width and height shift transformations are dependent on the range to either improve or hinder performance of the network in both cases. Only ranges over $40 \%$ produce a positive effect, up to 6.59 points, although statistical significance is not achieved in CVC-EndoSceneStill. If Kvasir-SEG is considered, these transformations improve the baseline if small ranges are used, but not significantly. Secondly, rotation and shear results are in all cases under the baseline threshold, reaching 4.43 points decrement in the performance for CVC-EndoSceneStill. On the contrary, these transforms improve performance on Kvasir-SEG in up to 3.41 points, being the greatest improvement in this dataset. Zooming the image has different results depending on whether it is zoom in or out in CVC-EndoSceneStill. Zooming in decreases the performance more than 3.5 points, while zooming out can improve results up to almost 5 points, but significance is not achieved. On the contrary, some ranges from both transforms improve performance in Kvasir-SEG although not significantly. In relation to flipping the image, when CVC-EndoSceneStill is considered, horizontally flipping the image hinders the performance but if flipping is vertical, then performance is increased. In both cases, changes are not significant. On the contrary, both transforms improve performance in Kvasir-SEG, also without statistical significance. Lastly, elastic deformation of the image leads to deterioration of performance of up to 4.45 points in CVC-EndoSceneStill, but improve performance in 1.72 points in Kvasir-SEG.

The second group of transformations modified the pixelvalue. On one hand, changes in brightness in CVCEndoSceneStill, regardless of modifying all channels equally or each channel independently, yield to a better performance of the model of more than 12 points, obtaining significant 

and ranges analysed in both datasets

\begin{tabular}{|c|c|c|c|c|c|c|c|}
\hline & \multirow[b]{2}{*}{ Transformation } & \multirow[b]{2}{*}{ Range } & \multirow[b]{2}{*}{$\begin{array}{l}\text { IoU on test set } \\
\text { CVC- } \\
\text { EndoSceneStill }\end{array}$} & \multirow[b]{2}{*}{$\begin{array}{l}\text { IoU on test set } \\
\text { Kvasir-SEG }\end{array}$} \\
\hline Transformation & Range & $\begin{array}{l}\text { IoU on test set } \\
\text { CVC- }\end{array}$ & $\begin{array}{l}\text { IoU on test set } \\
\text { Kvasir-SEG }\end{array}$ & & & & \\
\hline & & & & \multirow{13}{*}{ Zoom out } & $0.3,1$ & $58.41 \pm 10.40$ & $66.54 \pm 8.30$ \\
\hline \multirow{3}{*}{$\begin{array}{l}\text { None } \\
\text { Image-based } \\
\text { transforma- } \\
\text { tions }\end{array}$} & \multirow[t]{3}{*}{ N/A } & \multirow[t]{3}{*}{$59.10 \pm 9.35$} & \multirow[t]{3}{*}{$66.45 \pm 8.08$} & & $0.2,1$ & $57.71 \pm 10.34$ & $65.54 \pm 8.37$ \\
\hline & & & & & $0.1,1$ & $57.56 \pm 10.06$ & $64.05 \pm 8.51$ \\
\hline & & & & & $1,1.1$ & $58.70 \pm 9.12$ & $65.48 \pm 8.17$ \\
\hline \multirow[t]{9}{*}{ Width Shift } & $\pm 10 \%$ & $60.78 \pm 8.99$ & $67.09 \pm 7.96$ & & $1,1.2$ & $61.64 \pm 8.26$ & $66.25 \pm 8.09$ \\
\hline & $\pm 20 \%$ & $59.45 \pm 9.80$ & $67.34 \pm 8.06$ & & $1,1.3$ & $58.99 \pm 8.50$ & $65.88 \pm 8.03$ \\
\hline & $\pm 30 \%$ & $59.31 \pm 9.08$ & $66.28 \pm 8.22$ & & $1,1.4$ & $62.21 \pm 8.04$ & $66.13 \pm 7.98$ \\
\hline & $\pm 40 \%$ & $62.70 \pm 8.57$ & $65.94 \pm 8.22$ & & $1,1.5$ & $61.83 \pm 8.39$ & $66.56 \pm 7.86$ \\
\hline & $\pm 50 \%$ & $62.80 \pm 8.84$ & $66.23 \pm 8.09$ & & $1,1.6$ & $64.03 \pm 8.26$ & $67.38 \pm 7.80$ \\
\hline & $\pm 60 \%$ & $63.02 \pm 8.78$ & $66.90 \pm 7.86$ & & $1,1.7$ & $60.67 \pm 7.90$ & $67.38 \pm 7.83$ \\
\hline & $\pm 70 \%$ & $63.03 \pm 8.67$ & $66.82 \pm 7.87$ & & $1,1.8$ & $62.01 \pm 8.20$ & $67.97 \pm 7.63$ \\
\hline & $\pm 80 \%$ & $61.34 \pm 8.62$ & $65.41 \pm 7.92$ & & $1,1.9$ & $62.73 \pm 8.00$ & $67.91 \pm 7.57$ \\
\hline & $\pm 90 \%$ & $65.68 \pm 8.12 *$ & $65.82 \pm 7.72$ & & $1,2.0$ & $64.00 \pm 8.13$ & $67.97 \pm 7.64$ \\
\hline \multirow[t]{10}{*}{ Height shift } & $\pm 10 \%$ & $58.82 \pm 8.97$ & $67.00 \pm 7.98$ & \multirow{10}{*}{$\begin{array}{l}\text { Horizontal flip } \\
\text { Vertical flip } \\
\text { Elastic } \\
\text { deformation }\end{array}$} & True & $55.89 \pm 9.22$ & $67.57 \pm 8.11$ \\
\hline & $\pm 20 \%$ & $58.94 \pm 8.80$ & $67.12 \pm 8.08$ & & True & $59.54 \pm 8.90$ & $67.23 \pm 8.08$ \\
\hline & $\pm 30 \%$ & $61.81 \pm 8.74$ & $67.26 \pm 7.87$ & & 250,40 & $60.26 \pm 8.79$ & $65.92 \pm 8.19$ \\
\hline & $\pm 40 \%$ & $62.03 \pm 8.57$ & $67.23 \pm 7.80$ & & & & \\
\hline & $\pm 50 \%$ & $61.78 \pm 8.42$ & $67.17 \pm 7.89$ & & 500,40 & $59.17 \pm 9.31$ & $65.86 \pm 8.18$ \\
\hline & $\pm 60 \%$ & $60.21 \pm 8.64$ & $66.97 \pm 7.94$ & & 1000,40 & $57.93 \pm 9.12$ & $66.97 \pm 8.00$ \\
\hline & $\pm 70 \%$ & $61.55 \pm 8.46$ & $66.69+7.98$ & & 2000,40 & $57.83 \pm 8.86$ & $67.88 \pm 8.02$ \\
\hline & & $60.05+810$ & $00.09 \pm 1.98$ & & 3000,40 & $55.89 \pm 9.14$ & $68.17 \pm 8.00$ \\
\hline & $\pm 80 \%$ & $60.42 \pm 8.19$ & $66.26 \pm 7.94$ & & 4000,40 & $54.65 \pm 9.12$ & $66.96 \pm 8.20$ \\
\hline & $\pm 90 \%$ & $61.52 \pm 8.27$ & $67.06 \pm 7.58$ & & 5000,40 & $56.55 \pm 9.13$ & $65.17 \pm 8.36$ \\
\hline \multirow[t]{8}{*}{ Rotation } & $\pm 3^{\circ}$ & $57.74 \pm 9.37$ & $66.41 \pm 8.09$ & \multirow{4}{*}{$\begin{array}{l}\text { Pixel-based } \\
\text { transforma- } \\
\text { tions }\end{array}$} & 6000,40 & $55.90 \pm 9.37$ & $65.02 \pm 8.28$ \\
\hline & $\pm 6^{\circ}$ & $59.97 \pm 9.06$ & $65.61 \pm 8.16$ & & & & \\
\hline & $\pm 10^{\circ}$ & $55.40 \pm 9.75$ & $65.74 \pm 8.15$ & & & & \\
\hline & $\pm 15^{\circ}$ & $55.50 \pm 9.65$ & $67.03 \pm 8.10$ & & & & \\
\hline & $\pm 45^{\circ}$ & $54.66 \pm 9.62$ & $68.38 \pm 8.00$ & \multirow{4}{*}{$\begin{array}{l}\text { Brightness, all } \\
\text { channels } \\
\text { equally }\end{array}$} & \pm 25 & $59.89 \pm 84$ & $66.87 \pm 7.66$ \\
\hline & $\pm 90^{\circ}$ & $57.62 \pm 9.37$ & $69.86 \pm 7.79$ & & & & \\
\hline & $\pm 135^{\circ}$ & $58.60 \pm 9.49$ & $68.22 \pm 8.07$ & & & & \\
\hline & $\pm 180^{\circ}$ & $58.19 \pm 9.35$ & $68.78 \pm 8.10$ & & \pm 50 & $63.27 \pm 8.41$ & $66.22 \pm 7.74$ \\
\hline \multirow[t]{8}{*}{ Shear } & $\pm 3^{\circ}$ & $59.62 \pm 9.05$ & $66.24 \pm 8.11$ & \multirow{13}{*}{$\begin{array}{l}\text { Brightness, } \\
\text { each channel } \\
\text { independently }\end{array}$} & \pm 75 & $66.79 \pm 8.28 * *$ & $65.17 \pm 7.76$ \\
\hline & $\pm 6^{\circ}$ & $61.66 \pm 8.98$ & $67.00 \pm 8.02$ & & \pm 100 & $67.99 \pm 8.23 * *$ & $64.55 \pm 7.86$ \\
\hline & $\pm 10^{\circ}$ & $59.42 \pm 9.00$ & $67.32 \pm 7.90$ & & \pm 125 & $68.98 \pm 7.90 * * *$ & $63.95 \pm 7.87$ \\
\hline & $\pm 15^{\circ}$ & $57.91 \pm 9.10$ & $67.11 \pm 7.97$ & & \pm 150 & $70.07 \pm 7.75 * * *$ & $67.25 \pm 7.86$ \\
\hline & $\pm 45^{\circ}$ & $59.07 \pm 9.80$ & $6888+774$ & & \pm 175 & $68.32 \pm 7.74 * *$ & $67.70 \pm 7.88$ \\
\hline & $\pm 40^{\circ}$ & $56.38 \pm 9.3$ & $\begin{array}{l}\mathbf{0 0 . 0 0} \pm 1.14 \\
67.84 \pm 7.85\end{array}$ & & \pm 25 & $71.21 \pm 7.69 * * *$ & $67.85 \pm 7.84$ \\
\hline & $\pm 135^{\circ}$ & $55.22 \pm 9.37$ & $67.53 \pm 7.91$ & & & & \\
\hline & $\pm 180^{\circ}$ & $57.09 \pm 8.89$ & $67.67 \pm 7.90$ & & \pm 50 & $70.90 \pm 7.81 * * *$ & $68.28 \pm 7.78$ \\
\hline \multirow[t]{5}{*}{ Zoom in } & $0.9,1$ & $60.19 \pm 8.54$ & $66.71 \pm 8.08$ & & \pm 75 & $69.26 \pm 8.19 * * *$ & $68.91 \pm 7.60$ \\
\hline & $0.8,1$ & $59.98 \pm 8.53$ & $67.45 \pm 8.01$ & & \pm 100 & $69.07 \pm 8.26^{* * *}$ & $69.21 \pm 7.51$ \\
\hline & $0.7,1$ & $57.01 \pm 9.46$ & $67.56 \pm 8.24$ & & \pm 125 & $67.86 \pm 8.27^{* *}$ & $69.36 \pm 7.46$ \\
\hline & $0.6,1$ & $55.57 \pm 10.07$ & $68.54 \pm 8.14$ & & \pm 150 & $67.86 \pm 7.77^{* *}$ & $67.07 \pm 8.05$ \\
\hline & $0.5,1$ & $57.37 \pm 10.30$ & $68.80 \pm 8.25$ & & \pm 175 & $66.15 \pm 8.16^{*}$ & $68.39 \pm 7.65$ \\
\hline
\end{tabular}

Table 4 continued

Table 4 Mean and standard deviation of the mean for transformations 
Table 4 continued

\begin{tabular}{|c|c|c|c|}
\hline Transformation & Range & $\begin{array}{l}\text { IoU on test set } \\
\text { CVC- } \\
\text { EndoSceneStill }\end{array}$ & $\begin{array}{l}\text { IoU on test set } \\
\text { Kvasir-SEG }\end{array}$ \\
\hline \multirow{5}{*}{$\begin{array}{l}\text { Contrast, all } \\
\text { channels } \\
\text { equally }\end{array}$} & $0.8,1.2$ & $58.11 \pm 9.35$ & $66.89 \pm 7.98$ \\
\hline & $0.6,1.4$ & $61.55 \pm 8.76$ & $67.31 \pm 7.85$ \\
\hline & $0.4,1.6$ & $66.17 \pm 8.37^{*}$ & $67.92 \pm 7.56$ \\
\hline & $0.2,1.8$ & $68.38 \pm 8.06^{* *}$ & $68.16 \pm 7.63$ \\
\hline & $0.0,2.0$ & $60.54 \pm 9.43$ & $66.29 \pm 8.14$ \\
\hline \multirow{4}{*}{$\begin{array}{l}\text { Contrast, each } \\
\text { channel } \\
\text { independently }\end{array}$} & $0.8,1.2$ & $71.80 \pm 7.61 * * *$ & $67.68 \pm 7.95$ \\
\hline & $0.6,1.4$ & $71.70 \pm 7.62 * * *$ & $66.58 \pm 7.79$ \\
\hline & $0.4,1.6$ & $72.34 \pm 7.81^{* * *}$ & $66.45 \pm 7.63$ \\
\hline & $0.2,1.8$ & $70.54 \pm 7.97 * * *$ & $66.83 \pm 7.46$ \\
\hline \multicolumn{4}{|l|}{$\begin{array}{l}\text { Application- } \\
\text { based } \\
\text { transforma- } \\
\text { tions }\end{array}$} \\
\hline Specular lights & True & $59.64 \pm 9.06$ & $67.52 \pm 7.59$ \\
\hline \multirow[t]{7}{*}{ Blurry image } & 3 & $60.32 \pm 8.67$ & $66.14 \pm 8.01$ \\
\hline & 5 & $58.94 \pm 9.37$ & $65.54 \pm 8.01$ \\
\hline & 7 & $53.61 \pm 9.33$ & $64.86 \pm 8.05$ \\
\hline & 9 & $50.39 \pm 9.84^{* *}$ & $66.81 \pm 7.93$ \\
\hline & 11 & $51.24 \pm 10.02 *$ & $64.78 \pm 8.12$ \\
\hline & 13 & $52.21 \pm 9.75^{*}$ & $65.85 \pm 8.13$ \\
\hline & 15 & $48.41 \pm 10.32 * *$ & $64.91 \pm 8.13$ \\
\hline
\end{tabular}

Best value for each transformation is indicated in bold

Statistical differences between baseline and the particular case are identified with permutation test

${ }^{* * *} p$ value $<0.001 ; * * p$ value $<0.01 ; * p$ value $<0.05$

differences in all cases but two. Similarly, modifying the contrast reached an increment of 13.25 points with respect to the baseline, being this the greatest improvement in all transformations and ranges, and obtaining statistically significant differences for all ranges if channels are modified independently and two out of four if they are equally modified. This behaviour is not so strong in the Kvasir-SEG, while changing brightness and contrast do improve performance in some ranges, significance is not achieved.

Lastly, we analysed transformations based on specific problems of colonoscopy images: adding specular lights and blurring frames. In the first case, including specular lights increased performance in half point and one point regarding the baseline for each dataset, although significance is not achieved in any dataset. On the second case, blurring the image resulted on a significant decrement of up to 10.69 points when compared to the baseline in the case of CVCEndoScenestill, but only 1.59 points and no significance in Kvasir-SEG.

Based on these results, we have also analysed combinations of transformation for the different datasets. Results are included in Table 5 and Fig. 6. In all cases for CVCEndoSceneStill, the mean of these combinations is similar to the transformation with higher mean, but the distributions are improved as the 25 quartile is increased and the standard deviation is minimized. On the other hand, the combination of all image-based transformations hinders the performance, proving that more data augmentation is not always better [24], as only the two image-based transformations with higher mean obtain the best results.

\section{Discussion and conclusion}

Data augmentation is a useful tool to increase the number of training samples when the available dataset is scarce, a situation that is well-known when using medical images. The effect of different transformations usually applied in data augmentation for polyp segmentation has yet to be rigorously analysed. In this work, we have found that although image-based transformations are usually applied in the state of the art, pixel-based transformations produce better results for CVC-EndoSceneStill. These transformations modify the particular value of the pixel, so the model is invariant to colour information, which improves its generalization capacity. On the other hand, Kvasir-SEG benefits to a greater extent from the image-based transformations.

In the light of the results, four new groups of transformations can be established:

1. Transformations that always improve the performance in CVC-EndoScenStill and Kvasir-SEG: vertical flip, changes on brightness for each channel independently, changes on contrast (all channels equally and each channel independently) and inclusion of specular lights. All these transformations improve the performance over the baseline, although statistical significance is mainly found in changes of brightness and contrast in CVCEndoSceneStill.

2. Transformations that always hinder the performance in CVC-EndoScenStill and Kvasir-SEG: elastic deformation and blurry frames (mean filter). While blurry frames could be expected to minimize the performance as they reduce the details in the image, elastic deformation might have been expected to improve performance. Although blurry frames are a common situation during a live 


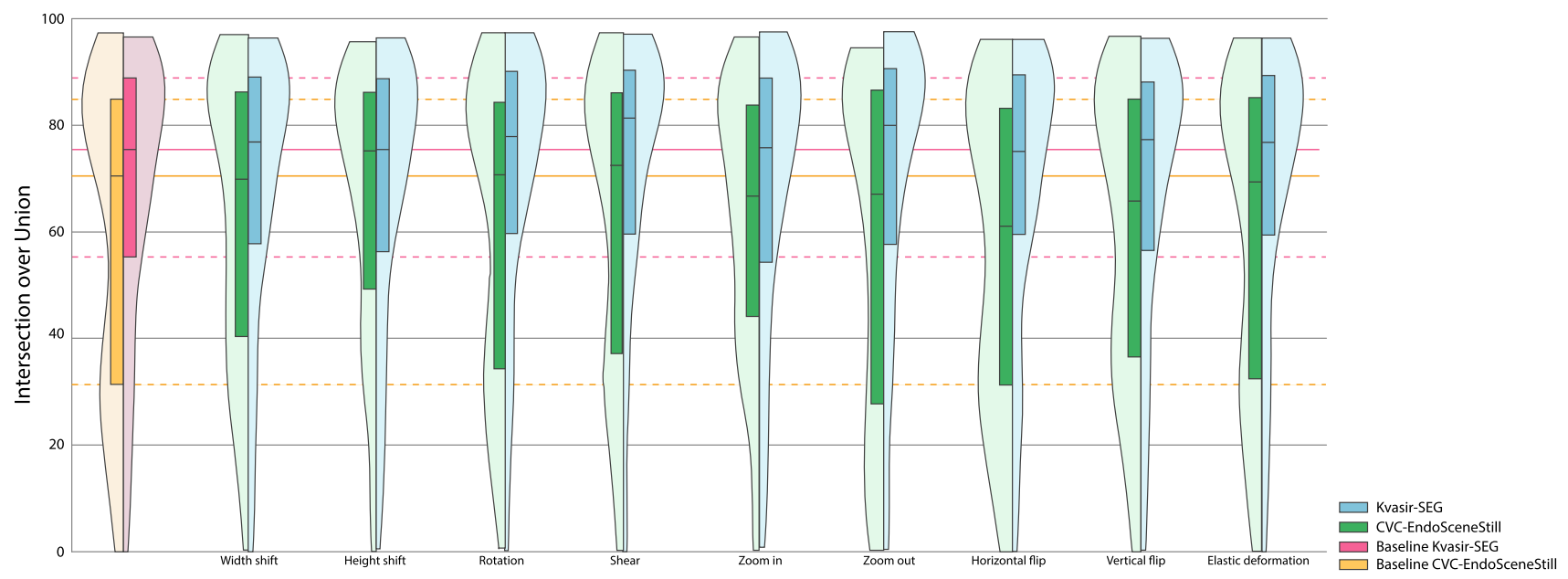

Fig. 3 Results for image-based transformations. Ranges with highest mean are shown for each transformation and dataset. Baselines of each dataset are included. Their median and quartiles are prolongated on the background for reference. For the CVC-EndoSceneStill: $\pm 90 \%$ width shift $\pm 40 \%$ height shift $\pm 6^{\circ}$ rotation, $\pm 45^{\circ}$ shear; 0.9 zoom in; 0.4 zoom out; $(250,40)$ elastic deformation. For the Kvasir-SEG: $\pm 20 \%$ width shift $\pm 30 \%$ height shift $\pm 90^{\circ}$ rotation, $\pm 45^{\circ}$ shear; 0.5 zoom in; 0.2 zoom out; $(3000,40)$ elastic deformation

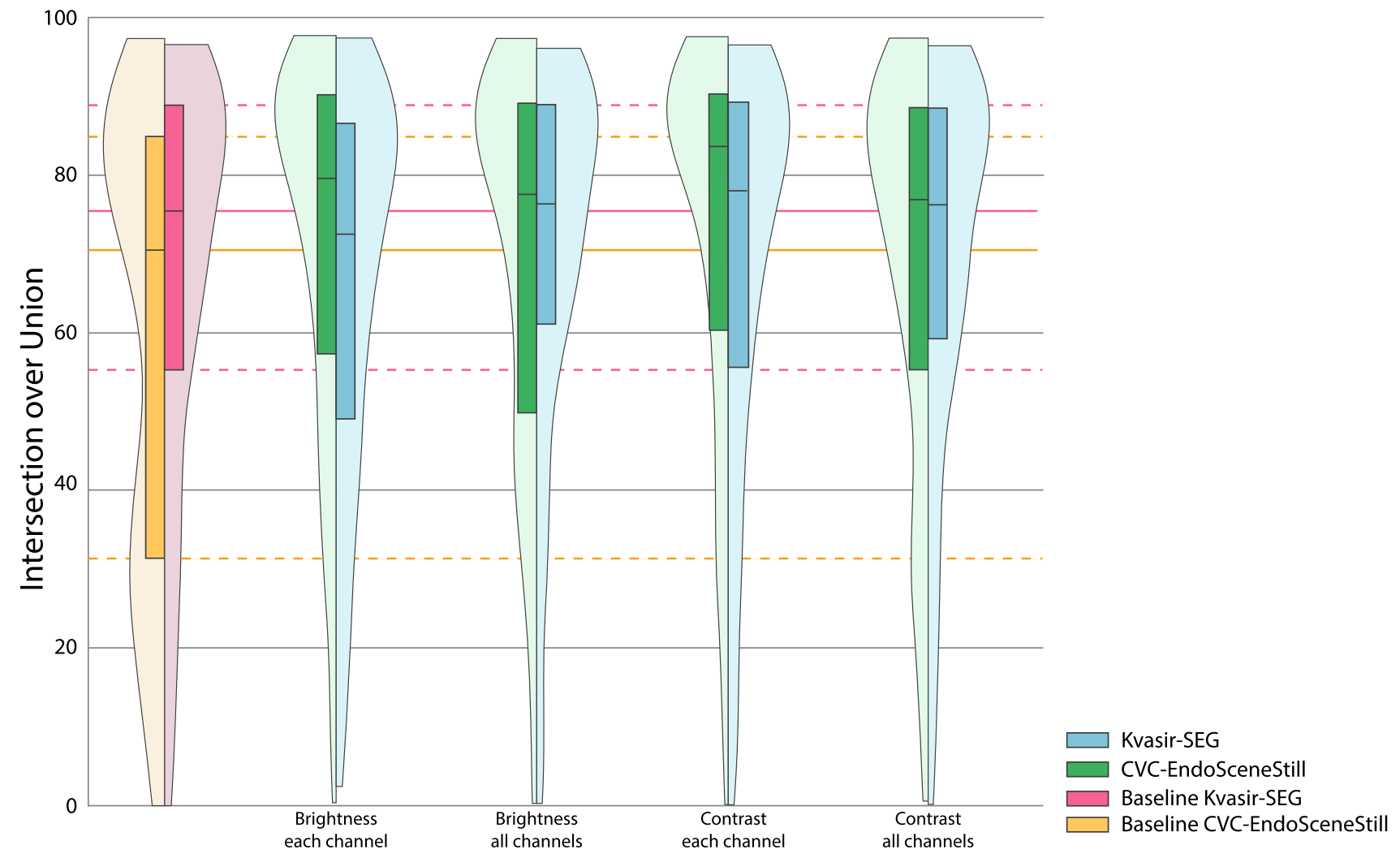

Fig. 4 Results for image-based transformations. Ranges with highest mean are shown for each transformation and dataset. Baselines of each dataset are included. Their median and quartiles are prolongated on the background for reference. For the CVC-EndoSceneStill: \pm 150 for brightness in all channels equally; \pm 25 for brightness in each chan- nel independently; (0.2-1.8) for contrast in all channels equally; and (0.4-1.6) for brightness in each channel independently. For the KvasirSEG: \pm 175 for brightness in all channels equally; \pm 125 for brightness in each channel independently; $(0.2-1.8)$ for contrast in all channels equally; and (0.8-1.2) for brightness in each channel independently 
Fig. 5 Results for problem-based transformations. Ranges with highest mean are shown for each transformation and dataset. Baselines of each dataset are included. Their median and quartiles are prolongated on the background for reference. For the CVC-EndoSceneStill: 3 for blurry images. For the Kvasir-SEG: 9 for blurry images

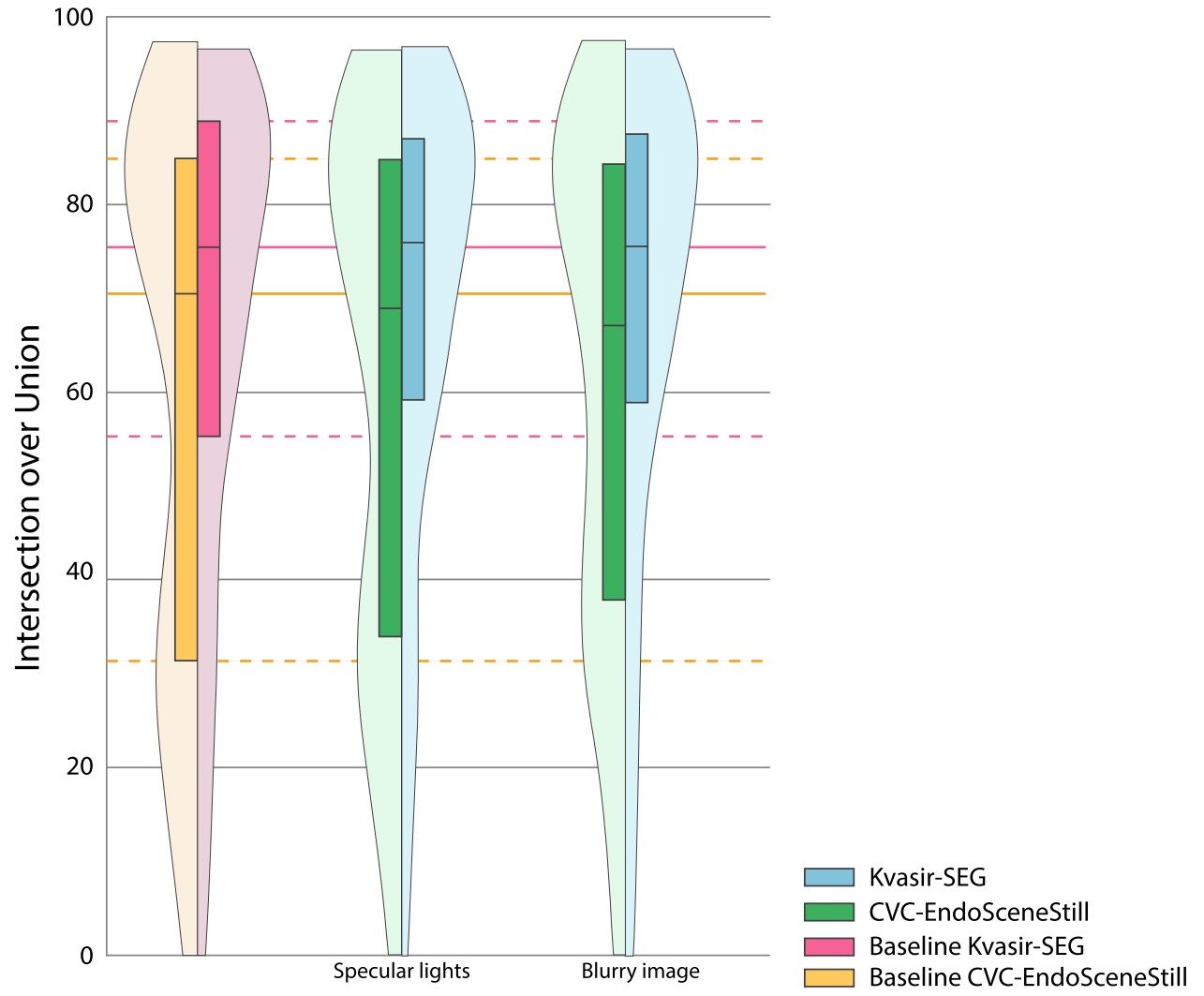

colonoscopy, the inclusion of mean filter as transformation for data augmentation does not improve the final performance of the model. This is probably explained by the use of databases, where frames are previously selected and not blurry frames are included.

3. Transformations whose effect on performance depends on the selected range in CVC-EndoScenStill and KvasirSEG: height and width shifts, as well as zoom in and out. In the first two cases, ranges over $40 \%$ do contribute to improve performance, while under the threshold either the transformation does not add improvement or decrement the performance. On the other hand, zoom behaviour also depends on the range. Smaller ranges of zoom in and larger ranges of zoom out improve the performance over the baseline, although not always significantly. One reason for the performance of the zoom in might be grounded on the low quality of the original images, resulting in blurry zoomed images. Therefore, when using them for data augmentation, it is recommended to carefully check whether the range is suitable or not.

4. Transformations whose effect on performance depends on the dataset, CVC-EndoScenStill or Kvasir-SEG: This relates mainly to rotation, shear and changes on bright- ness for all channels equally, and, to a lesser extent, horizontal flip. This might be due to differences in polyp size, void area, brightness and contrast in the images of the two datasets.

In summary, CVC-EndoSceneStill is more prone to benefits of data augmentation if pixel-based transformations are used, as the histogram is flatter, and images are darker than in Kvasir-SEG. On the contrary, image-based transformations appear to be more suitable in Kvasir-SEG, where the void area is smaller, and the polyp occupy a greater area of the valid image. Lastly, problem-based transformations behave similarly in both datasets, as they are rooted on the endoscopic image acquisition. It is also important to mention that the baseline of Kvasir-Seg showed already a better performance than CVC-EndosSceneStill, giving less room for improvement to data augmentation.

There are different approaches to overcome the scarce labelled datasets in medical imaging. On the one side, and in order to increase the size of the training set, a first approach would be to increase the number of annotated samples by experts. In this regard, efforts are been focused on developing tools which facilitates the manual annotation of images, such as GTCreatorTool [22], which is a flexible annotation 
Table 5 Mean and standard deviation of combinations analysed

\begin{tabular}{|c|c|c|c|c|}
\hline & \multicolumn{2}{|l|}{ CVC-EndoSceneStill } & \multicolumn{2}{|l|}{ Kvasir-SEG } \\
\hline & Transformations & IoU on test set & Transformations & IoU on test set \\
\hline Baseline & None & $59.10 \pm 9.35$ & None & $66.45 \pm 8.08$ \\
\hline $\begin{array}{l}\text { Transformation and range with } \\
\text { highest mean for each one of } \\
\text { the three types of transforms }\end{array}$ & $\begin{array}{l}\text { Width at } \pm 90 \% \\
\text { Change of contrast: each channel } \\
\text { independently, with range }[0.4 \text {, } \\
\text { 1.6] } \\
\text { Inclusion of specular lights }\end{array}$ & $72.30 \pm 7.26^{* * *}$ & $\begin{array}{l}90^{\circ} \text { rotation } \\
\text { Change of brightness: each } \\
\text { channel independently, with } \\
\text { range } \pm 125 \\
\text { Inclusion of specular lights }\end{array}$ & $65.53 \pm 7.98$ \\
\hline $\begin{array}{l}\text { Range with highest mean of the } \\
\text { image-based transformations, } \\
\text { provided that they improve the } \\
\text { baseline result }\end{array}$ & $\begin{array}{l}\text { Width at } \pm 90 \% \\
\text { Height at } \pm 40 \% \\
\text { Zoom with range }[1,1.6] \\
\text { Vertical flip }\end{array}$ & $65.19 \pm 7.81 *$ & $\begin{array}{l}\text { Width at } \pm 20 \% \\
\text { Height at } \pm 30 \% \\
90^{\circ} \text { rotation } \\
45^{\circ} \text { shear } \\
\text { Zoom with range }[0.5,1] \\
\text { Vertical flip } \\
\text { Horizontal flip } \\
\text { Elastic deformation, with values } \\
\quad(3000,40)\end{array}$ & $57.97 \pm 9.21 * *$ \\
\hline $\begin{array}{l}\text { The two transformations with } \\
\text { higher mean }\end{array}$ & $\begin{array}{l}\text { Change of contrast: each channel } \\
\text { independently, with range [0.4, } \\
\text { 1.6] } \\
\text { Change of brightness: each } \\
\text { channel independently, with } \\
\text { range } \pm 25\end{array}$ & $70.50 \pm 7.69 * * *$ & $45^{\circ}$ shear & $69.24 \pm 7.85$ \\
\hline
\end{tabular}

Statistical differences between baseline and combination are identified with permutation test

${ }^{* * *} p$ value $<0.001 ; * * p$ value $<0.01 ; * p$ value $<0.05$

tool which minimizes annotation time and allows for sharing annotations among experts. Beyond the transformations analysed in this paper, other alternatives would be to add polyps in nonpolypoid samples [41] or more advances approaches such as emulating data augmentation during learning by the image generation through a hetero-encoder [42]. On the other hand, it would be possible to explore alternatives to supervised training, which already seems to provide good results with self-supervised learning [43] or similarity-based active Learning [44].

There are limitations in this study that must be acknowledged. Ideally, it would be necessary to independently analyse all combinations. Since that would mean almost 6 million experiments, alternatives such as AutoAugment [7] or Smart Augmentation [10] would be more suitable for identification of the best combination of transformations. Another possibility could be the application of Bayesian methods [45] or coordinate ascent optimization [46, 47] taking the optimal setting of each transform to identify the best combination. Future work should place emphasis on applying any of these alternatives to the particular field of polyp segmentation. Another limitation is the fact that the experiments have not pursued the best model, so training has been stopped at 15 epochs. It might be possible that with a more extensive training some of the transformations could have showed better results. Nevertheless, 15 epochs is enough training to establish the tendency of the model performance when finetuning it with a small database.

Further research is also possible in this line of work. Future works might focus on the effect of data augmentation on other segmentation approaches, such as the fuzzy C-mean clustering, which has shown good preliminary results on the Kvasir-SEG database [20].

In conclusion, this study shows that different transformations and ranges lead to differences in model performance. Despite not being so frequent as the other types, pixel-based transformations show a great potential to improve polyp segmentation. Augmenting colour variability when training the model allows for a better generalization of the model resulting in better prediction. On the other hand, image-based transformations and their ranges should be carefully selected to not hinder the model performance and obtain the expected benefits of data augmentation. 


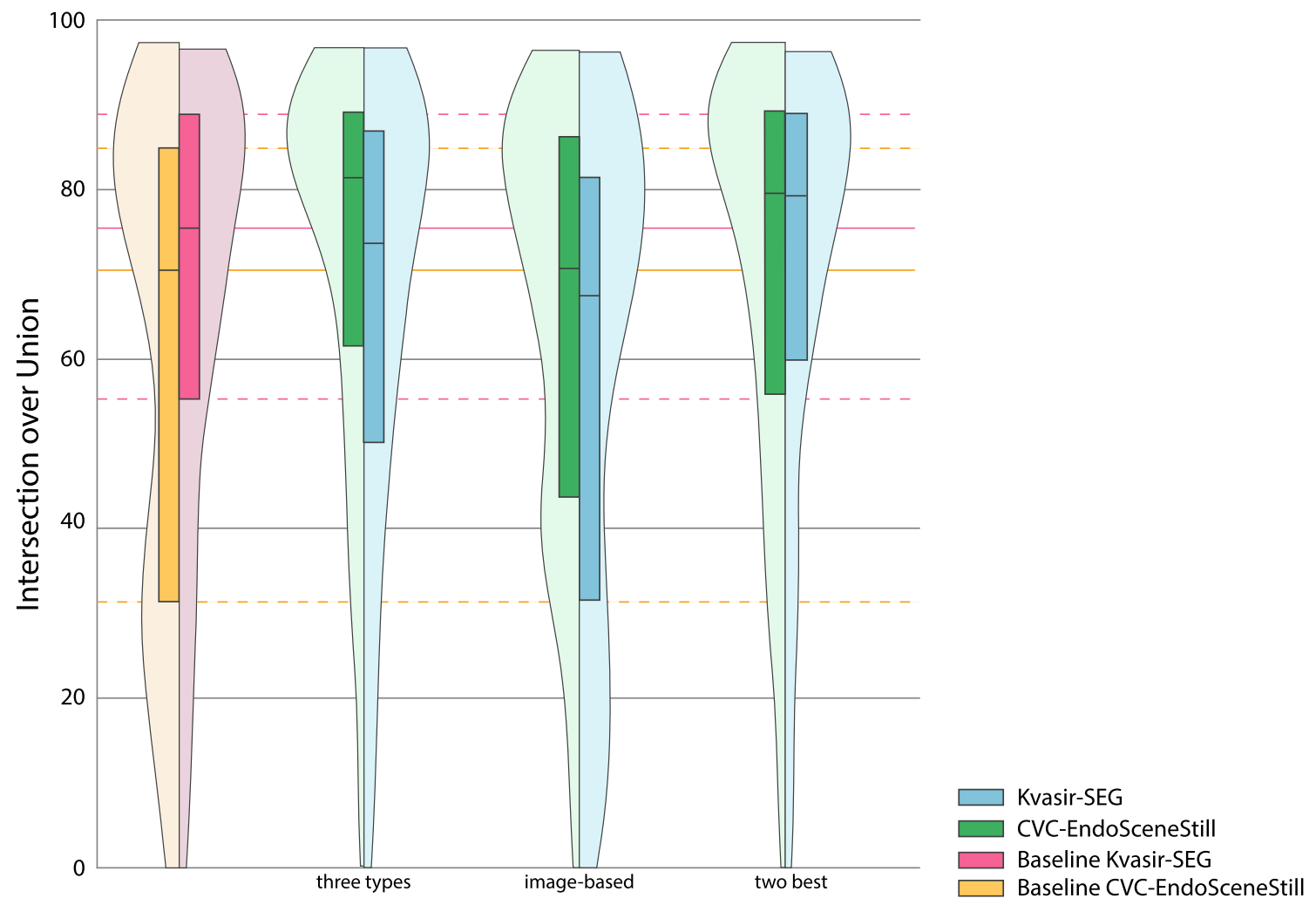

Fig. 6 Results for combination of transformations. Baselines of each dataset are included. Their median and quartiles are prolongated on the background for reference. Combination of the transformation and range with highest mean for each one of the three types of transforms for each dataset. For CVC-EndoSceneStill: width at $\pm 90 \%$, change of contrast: each channel independently, with range $[0.4,1.6]$, and inclusion of specular lights. For Kvasir-SEG: $90^{\circ}$ rotation, change of brightness: each channel independently, with range \pm 125 , and inclusion of specular lights. Combination of the range with highest mean of the image- based transformations, provided that they improve the baseline result. For CVC-EndoSceneStill: width at $\pm 90 \%$, height at $\pm 40 \%$, zoom with range $[1,1.6]$, and vertical flip. For Kvasir-SEG: width at $\pm 20 \%$, height at $\pm 30 \%, 90^{\circ}$ rotation, $45^{\circ}$ shear, zoom with range [0.5, 1], vertical flip, horizontal flip, and elastic deformation, with values $(3000,40)$. Combination of the two transformations with higher mean. For CVCEndoSceneStill: change of contrast: each channel independently, with range $[0.4,1.6]$ and change of brightness: each channel independently, with range \pm 25 . For Kvasir-SEG: $90^{\circ}$ rotation and $45^{\circ}$ shear
Acknowledgements The authors would like to thank Agueda Azpeitia and Juan Carlos Gómez-Blanco for their statistically significant help.

Funding This work was partially supported by PICCOLO project. This project has received funding from the European Union's Horizon2020 research and innovation programme under Grant Agreement No 732111. The sole responsibility of this publication lies with the author. The European Union is not responsible for any use that may be made of the information contained therein.

\section{Compliance with ethical standards}

Conflict of interest The authors declare that they have no conflict of interest.

Ethical approval This article does not contain any studies with human participants or animals performed by any of the authors.

Informed consent This article does not contain patient data.
Open Access This article is licensed under a Creative Commons Attribution 4.0 International License, which permits use, sharing, adaptation, distribution and reproduction in any medium or format, as long as you give appropriate credit to the original author(s) and the source, provide a link to the Creative Commons licence, and indicate if changes were made. The images or other third party material in this article are included in the article's Creative Commons licence, unless indicated otherwise in a credit line to the material. If material is not included in the article's Creative Commons licence and your intended use is not permitted by statutory regulation or exceeds the permitted use, you will need to obtain permission directly from the copyright holder. To view a copy of this licence, visit http://creativecomm ons.org/licenses/by/4.0/.

\section{References}

1. LeCun Y, Bengio Y, Hinton G (2015) Deep learning. Nature 521:436-444. https://doi.org/10.1038/nature14539

2. Biswas M, Kuppili V, Saba L, Edla DR, Suri HS, Cuadrado-Godia E, Laird JR, Marinhoe RT, Sanches JM, Nicolaides A, Suri JS 
(2019) State-of-the-art review on deep learning in medical imaging. Front Biosci 24:380-406. https://doi.org/10.2741/4725

3. Ker J, Wang L, Rao J, Lim T (2018) Deep learning applications in medical image analysis. IEEE Access 6:9375-9389. https://doi. org/10.1109/ACCESS.2017.2788044

4. Litjens G, Kooi T, Bejnordi BE, Setio AAA, Ciompi F, Ghafoorian M, van der Laak JAWM, van Ginneken B, Sánchez CI (2017) A survey on deep learning in medical image analysis. Med Image Anal 42:60-88. https://doi.org/10.1016/j.media.2017.07.005

5. Medela A, Picon A, Saratxaga CL, Belar O, Cabezón V, Cicchi R, Bilbao R, Glover B (2019) Few shot learning in histopathological images: reducing the need of labeled data on biological datasets. In: 2019 IEEE 16th international symposium on biomedical imaging (ISBI). https://doi.org/https://doi.org/10.1109/ISBI.2019.8759182

6. Shorten C, Khoshgoftaar TM (2019) A survey on image data augmentation for deep learning. J Big Data. https://doi.org/10.1186/s4 0537-019-0197-0

7. Cubuk ED, Zoph B, Mane D, Vasudevan V, Le QV (2018) AutoAugment: learning augmentation policies from data. arXiv. https://arxiv.org/abs/1805.09501. Accessed 5 Jan 2020

8. Jung AB, Wada K, Crall J, Tanaka S, Graving J,Yadav S, Banerjee J, Vecsei G, Kraft A, Borovec J, Vallentin C, Zhydenko S, Pfeiffer K, Cook B, Fernández I, Chi-Hung W, Ayala-Acevedo A, Meudec R, Laporte M (2019) Imgaug. https://github.com/aleju/ imgaug. Accessed 5 Jan 2020

9. Casado-García Á, Domínguez C, García-Domínguez M, Heras J, Mata E, Pascual V (2019) CLoDSA: a tool for augmentation in classification, localization, detection, semantic segmentation and instance segmentation tasks. BMC Bioinform 20:1-14. https://doi. org/10.1186/s12859-019-2931-1

10. Lemley J, Bazrafkan S, Corcoran P (2017) Smart augmentation learning an optimal data augmentation strategy. IEEE Access 5:5858-5869. https://doi.org/10.1109/ACCESS.2017.2696121

11. Okafor E, Schomaker L, Wiering MA (2018) An analysis of rotation matrix and colour constancy data augmentation in classifying images of animals augmentation in classifying images of animals. J Inf Telecommun. https://doi.org/10.1080/24751839.2018.14799 32

12. Shijie J, Ping W, Peiyi J, Siping H (2017) Research on data augmentation for image classification based on convolution neural networks. In: 2017 Chinese automation congress (CAC). pp 4165-4170

13. Wang J, Perez L (2017) The effectiveness of data augmentation in image classification using deep learning. https://arxiv.org/abs/171 2.04621

14. Zhang C, Tavanapong W, Wong J, de Groen PC, Oh JH (2017) Real data augmentation for medical image classification. In: Intravascular imaging and computer assisted stenting, and large-scale annotation of biomedical data and expert label synthesis. pp 67-76. https://doi.org/https://doi.org/10.1007/978-3-319-67534-3_8

15. Ma R, Tao P, Tang H (2019) Optimizing data augmentation for semantic segmentation on small-scale dataset. In: Proceedings of the 2nd international conference on control and computer vision-ICCCV 2019. pp 77-81

16. Urban G, Tripathi P, Alkayali T, Mittal M, Jalali F, Karnes W, Baldi P (2018) Deep learning localizes and identifies polyps in real time with $96 \%$ accuracy in screening colonoscopy. Gastroenterology 155:1069-1078.e8. https://doi.org/10.1053/j.gastro.2018.06.037

17. Wang P, Berzin TM, Glissen Brown JR, Bharadwaj S, Becq A, Xiao X, Liu P, Li L, Song Y, Zhang D, Li Y, Xu G, Tu M, Liu X (2019) Real-time automatic detection system increases colonoscopic polyp and adenoma detection rates: a prospective randomised controlled study. Gut. https://doi.org/10.1136/gutjnl-2 018-317500

18. Alagappan M, Brown JRG, Mori Y, Berzin TM (2018) Artificial intelligence in gastrointestinal endoscopy: the future is almost here.
World J Gastrointest Endosc 10:239-249. https://doi.org/10.4253/ wjge.v10.i10.239

19. Vázquez D, Bernal J, Sánchez FJ, Fernández-Esparrach G, López AM, Romero A, Drozdzal M, Courville A (2017) A benchmark for endoluminal scene segmentation of colonoscopy images. J Healthc Eng. https://doi.org/10.1155/2017/4037190

20. Jha D, Smedsrud PH, Riegler MA, Halvorsen P, Johansen HD, de Lange T, Johansen D (2020) Kvasir-SEG: a segmented polyp dataset. In: Proceedings of the international conference on multimedia modeling (MMM)

21. Angermann Q, Bernal J, Sánchez-Montes C, Hammami M, Fernández-Esparrach G, Dray X, Romain O, Sánchez FJ, Histace A (2017) Real-time polyp detection in colonoscopy videos: a preliminary study for adapting still frame-based methodology to video sequences analysis. In: International journal of computer assisted radiology and surgery. https://hal.archives-ouvertes.fr/hal-014886 57

22. Bernal J, Histace A, Masana M, Angermann Q, Sánchez-Montes C, Rodríguez de Miguel C, Hammami M, García-Rodríguez A, Córdova H, Romain O, Fernández-Esparrach G, Dray X, Sánchez FJ (2019) GTCreator: a flexible annotation tool for image-based datasets. Int J Comput Assist Radiol Surg 14:191-201. https://doi. org/10.1007/s11548-018-1864-X

23. Yu J, Pan H, Yin Q, Bian X, Cui Q (2019) Fully convolutional densenets for polyp segmentation in colonoscopy. In: 2019 IEEE 35th international conference on data engineering workshops (ICDEW), Macao, Macao, 2019. pp 306-311. https://doi.org/https://doi.org/10.1109/ICDEW.2019.00010

24. Shin Y, Qadir HA, Aabakken L, Bergsland J, Balasingham I (2018) Automatic colon polyp detection using region based deep CNN and post learning approaches. IEEE Access 6:40950-40962. https:// doi.org/10.1109/ACCESS.2018.2856402

25. Bernal J, Sánchez FJ, Rodríguez de Miguel C, FernándezEsparrach G (2015) Building up the future of colonoscopy? A synergy between clinicians and computer scientists. In: Screening for colorectal cancer with colonoscopy. InTech

26. Guo X, Zhang N, Guo J, Zhang H, Hao Y, Hang J (2019) Automated polyp segmentation for colonoscopy images: a method based on convolutional neural networks and ensemble learning. Med Phys 46:5666-5676. https://doi.org/10.1002/mp.13865

27. Kang J, Gwak J (2019) Ensemble of instance segmentation models for polyp segmentation in colonoscopy images. IEEE Access 7:26440-26447. https://doi.org/10.1109/ACCESS.2019.2900672

28. Akbari M, Mohrekesh M, Nasr-Esfahani E, Soroushmehr SMR, Karimi N, Samavi S, Najarian K (2018) Polyp segmentation in colonoscopy images using fully convolutional network. arXiv. https://arxiv.org/abs/1802.00368

29. Brandao P, Zisimopoulos O, Mazomenos E, Ciuti G, Bernal J, Visentini-Scarzanella M, Menciassi A, Dario P, Koulaouzidis A, Arezzo A, Hawkes DJ, Stoyanov D (2018) Towards a computedaided diagnosis system in colonoscopy: automatic polyp segmentation using convolution neural networks. J Med Robot Res 3:1-13. https://doi.org/10.1142/S2424905X18400020

30. Wichakam I, Panboonyuen T, Udomcharoenchaikit C (2018) Realtime polyps segmentation for colonoscopy video frames using compressed fully convolutional network. In: Multimedia modeling. MMM 2018. Lecture notes in computer science, vol 10704. pp 393-404

31. Wickstrøm K, Kampffmeyer M, Jenssen R (2018) Uncertainty modeling and interpretability in convolutional neural networks for polyp segmentation. In: 2018 IEEE international workshop on machine learning for signal processing

32. Bardhi O, Member I, Sierra-Sosa D, Garcia-Zapirain B, Elmaghraby A (2017) Automatic colon polyp detection using Convolutional Encoder-Decoder model. In: 2017 IEEE international symposium on signal processing and information technol- 
ogy (ISSPIT). pp 445-448. https://doi.org/https://doi.org/10.1109/ ISSPIT.2017.8388684

33. Li Q, Yang G, Chen Z, Huang B, Chen L, Xu D, Zhou X, Zhong, Zhang H, Wang T (2017) Colorectal polyp segmentation using a fully convolutional neural network. In: 2017 10th international congress on image and signal processing, biomedical engineering and informatics (CISP-BMEI). pp 1-5. https://doi.org/https://doi. org/10.1109/CISP-BMEI.2017.8301980

34. Abaza A, Harrison MA, Bourlai T (2012) Quality metrics for practical face recognition. In Proceedings of the proceedings of the 21st international conference on pattern recognition (ICPR2012). pp 3103-3107

35. Tripathi AK, Mukhopadhyay S, Dhara AK (2011) Performance metrics for image contrast. In Proceedings of the 2011 international conference on image information processing. https://doi.org/https://doi.org/10.1109/ICIIP.2011.6108900

36. Ronneberger O, Fischer P, Brox T (2015) U-Net: convolutional networks for biomedical image segmentation. In: Navab N, Hornegger J, Wells WM, Frangi AF (eds) Medical image computing and computer-assisted intervention-MICCAI 2015. Lecture notes in computer science, vol 9351. Springer, pp 234-241

37. Chollet F (2015) Keras. https://github.com/keras-team/keras

38. Abadi M, Agarwal A, Barham P, Brevdo E, Chen Z, Citro C, Corrado GS, Davis A, Dean J, Devin M, Ghemawat S, Goodfellow I, Harp A, Irving G, Isard M, Jia Y, Jozefowicz R, Kaiser L, Kudlur M, Levenberg J, Mané D, Monga R, Moore S, Murray D, Olah C, Schuster M, Shlens J, Steiner B, Sutskever I, Talwar K, Tucker P, Vanhoucke V, Vasudevan V, Viégas F, Vinyals O, Warden P, Wattenberg M, Wicke M, Yu Y, Zheng X (2015) TensorFlow: large-scale machine learning on heterogeneous distributed systems. https:// arxiv.org/abs/1603.04467. Accessed 05 Jan 2020

39. Shvets AA, Rakhlin A, Kalinin AA, Iglovikov VI (2018) Automatic instrument segmentation in robot-assisted surgery using deep learning. In: 2018 17th IEEE international conference on machine learning and applications ICMLA 2018. pp 624-628

40. Wilcox RR (2003) Comparing two independent groups. In: Wilcox RR (ed) Applying Contemporary Statistical Techniques. Elsevier Science, Berlin, pp 237-284
41. De Almeida Thomaz V, Sierra-Franco CA, Raposo AB (2019) Training data enhancements for robust polyp segmentation in colonoscopy images. In: Proceedings-IEEE symposium on computer-based medical systems. pp 192-197

42. Hwang M, Wang D, Jiang WC, Pan X, Fu D, Hwang KS, Ding K (2019) An adaptive regularization approach to colonoscopic polyp detection using a cascaded structure of encoder-decoders. Int $\mathbf{J}$ Fuzzy Syst 21:2091-2101. https://doi.org/10.1007/s40815-019-00 694-y

43. Ross T, Zimmerer D, Vemuri A, Isensee F, Wiesenfarth M, Bodenstedt S, Both F, Kessler P, Wagner M, Müller B, Kenngott H, Speidel S, Kopp-Schneider A, Maier-Hein K, Maier-Hein L (2018) Exploiting the potential of unlabeled endoscopic video data with self-supervised learning. Int J Comput Assist Radiol Surg 13:925-933. https://doi.org/10.1007/s11548-018-1772-0

44. Zhang C, Tavanapong W, Kijkul G, Wong J, de Groen PC, Oh JH (2018) Similarity-based active learning for image classification under class imbalance. In: Proceedings of the IEEE international conference on data mining, ICDM 2018-November. pp 1422-1427. https://doi.org/https://doi.org/10.1109/ICDM.2018.00196

45. Tran T, Pham T, Carneiro G, Palmer L, Reid I (2017) A Bayesian data augmentation approach for learning deep models. In: 31st conference on neural information processing systems (NIPS 2017)

46. Saltiel D, Benhamou E (2018) Feature selection with optimal coordinate ascent (OCA). SSRN Electron J. https://doi.org/10.2139/ ssrn. 3293503

47. Zarshenas A, Suzuki K (2016) Binary coordinate ascent: an efficient optimization technique for feature subset selection for machine learning. Knowl Based Syst 110:191-201. https://doi.org/ 10.1016/j.knosys.2016.07.026

Publisher's Note Springer Nature remains neutral with regard to jurisdictional claims in published maps and institutional affiliations. 